\title{
Mixed convection over a horizontal circular cylinder embedded in porous medium immersed in a nanofluid with convective boundary conditions at lower stagnation point: A numerical solution
}

\author{
Norhafizah Md Sarif ${ }^{1, *}$, Mohd Zuki Salleh ${ }^{1}$, and Roslinda Nazar $^{2}$ \\ ${ }^{1}$ Faculty of Industrial Sciences \& Technology, Universiti Malaysia Pahang, Lebuhraya Tun Razak, 26300 \\ Gambang,Kuantan,Pahang, Malaysia \\ ${ }^{2}$ School of Mathematical Sciences, Faculty of Science and Technology, Universiti Kebangsaan \\ Malaysia, 43600 UKM Bangi, Selangor, Malaysia
}

\begin{abstract}
This study aims to examine the effect of governing parameters on the flow and heat transfer of the steady mixed convection flow embedded in porous medium with convective boundary conditions. The resulting system of nonlinear partial differential equations is solved numerically. The special case at the lower stagnation point of the cylinder is observed and the case where bottom surface of the cylinder is heated by convection from hot fluids is considered. Numerical solutions are obtained for the velocity, temperature and nanoparticle volume fraction profiles for two values of governing parameters namely convective parameter $\gamma$ and Lewis number Le. It is found that as the convective parameter $\gamma$ increases, velocity profile, temperature and nanoparticle volume fraction profile also increases.
\end{abstract}

\section{Introduction}

The influence of thermal conductivities in many fields of applications such as energy production, supply to electronic and transportation contributes to an accelerating interest in the development of nanofluids. However, the performances of industrial devices are limited due to the low thermal conductivity of convectional fluid. Thermal conductivity can be improved by suspending metallic nanoparticles within it (Tiwari \& Das [1]). The process of suspended the metallic, nonmetallic or polymeric nano-sized particle in a fluids are called nanofluid.

Buongiorno [2] conducted a study of convective transport in nanofluid, focusing on the heat transfer enhancement. Based on Buongiorno's exploration on nanofluid, the study on the flow in porous medium filled by nanofluid has garnered considerable attention. Nield \& Kuznetsov [3] pioneered the study of porous medium filled in nanofluid by presenting the influence of nanoparticle on free convection past a vertical plate, using Buongiorno's model. This problem is originally extend from the classical problem of porous medium studied by Cheng and Minkowycz [4].

\footnotetext{
*Corresponding author: norhafizah@ump.edu.my
} 
Further, as a continuation from Nield \& Kuznetsov [3] paper, Nield \& Kuznetsov [5] and Kuznetsov \& Nield [6] presented free convection of a porous medium immersed by a nanofluid past a vertical plate. Assumption has been made that nanoparticles are suspended in the nanofluid using either surfactant technology or surface charge. Furthermore, Khan \& Aziz [7] extended the previous work by examining the doublediffusion in a porous medium saturated with a nanofluid. An analytical solution of nanofluid in porous medium using Laplace transform method has been discussed by Hussanan et. al [8]

Notwithstanding, very limited work has been undertaken to investigate mixed convection flow past cylinders immersed in nanofluid. Such types of problems are crucial in many engineering devices and in the processes involving high temperatures. In 2014, Tham et. al [9] have examined the mixed convection boundary layer flow over a circular cylinder in a nanofluid with a constant wall temperature. Recently, Sarif et. al [10] analyzed the convection flow over a horizontal circular cylinder using Tiwari \& Das model using convective boundary conditions.

It is worth mentioning that in this study, we have followed closely the work of Tham et. al [9] where we extended their problem for the case of convective boundary condition where the bottom surface of the cylinder is heated by convection from hot fluids. The resulting layer boundary conditions are solved numerically using an implicit finite-difference scheme, known as Keller- box method. The vast number of published papers on various flow problems has successfully applied the Keller-box method in solving many flow problems. Also, many books describing the Keller-box method can be found in publications by $\mathrm{Na}$ [11] and Cebeci \& Bradshaw [12]. Particular efforts have been focused of temperature, velocity and nanoparticles volume fraction profile near the stagnation point of the cylinder. The effects of these parameters are presented through figures.

\section{Mathematical formulation}

The steady mixed convection boundary layer flow over horizontal circular cylinder of radius $a$ embedded in a porous medium immersed in water-based nanofluid is considered. The bottom of the cylinder is heated by convection from the hot fluid at temperature $T_{f}$ which yields a heat transfer coefficient $h_{f}$. Fig. 1 shows the physical model and coordinate system of this problem.

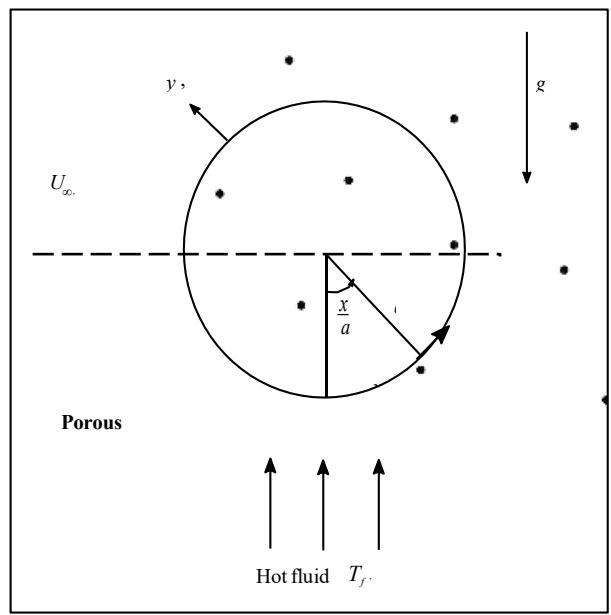

Fig. 1. Physical model and coordinate system. 
The governing equations for the present problem are (see Tham et. al [9]

$$
\begin{gathered}
\frac{\partial u}{\partial x}+\frac{\partial v}{\partial y}=0 \\
\frac{\partial u}{\partial y}=\left(\frac{\partial \theta}{\partial y}-N r \frac{\partial \phi}{\partial y}\right) \lambda \sin x \\
u \frac{\partial \theta}{\partial x}+v \frac{\partial \theta}{\partial y}=\frac{\partial^{2} \theta}{\partial y^{2}}+N_{b} \frac{\partial \theta}{\partial y} \frac{\partial \phi}{\partial y}+N_{t}\left(\frac{\partial \theta}{\partial y}\right)^{2} \\
\operatorname{Le}\left(u \frac{\partial \phi}{\partial x}+v \frac{\partial \phi}{\partial y}\right)=\frac{\partial^{2} \phi}{\partial y^{2}}+\left(\frac{N_{t}}{N_{b}}\right) \frac{\partial^{2} \theta}{\partial y^{2}}
\end{gathered}
$$

subject to boundary conditions

$$
\begin{aligned}
& v(x, y)=0, \theta^{\prime}(x, y)=-\gamma(1-\theta(x, y)), \quad \phi(x, y)=1 \text { at } y=0,0 \leq x \leq \pi \\
& u(x, y) \rightarrow u_{e}(x), \theta(x, y) \rightarrow 0, \phi(x, y) \rightarrow 0 \quad \text { as } y \rightarrow \infty, 0 \leq x \leq \pi
\end{aligned}
$$

Here $\lambda$ is the mixed convection parameter, $\gamma$ is the convective parameter, $L e$ is the traditional Lewis number, $N_{r}, N_{b}, N_{t}$ is the buoyancy, Brownian motion and thermophoresis parameters, respectively. These parameters are defined as

$$
\begin{aligned}
& \lambda=\frac{R a}{P e}, \quad L e=\frac{\alpha_{m}}{\varepsilon D_{B}}, \quad N_{r}=\frac{\left(\rho_{p}-\rho_{f_{\infty}}\right)\left(C_{w}-C_{\infty}\right)}{\rho_{f_{\infty}}\left(1-C_{\infty}\right) \beta\left(T_{f}-T_{\infty}\right)} \\
& N_{b}=\frac{\tau D_{B} \nabla\left(C_{w}-C_{\infty}\right)}{\alpha_{m}}, \quad N_{t}=\frac{\tau D_{T} \nabla\left(T_{f}-T_{\infty}\right)}{\alpha_{m} T_{\infty}}, \quad \gamma=\frac{h_{f} P e}{k a}
\end{aligned}
$$

Integrating equation (2) and using boundary conditions equation (5), we obtain

$$
u=\sin x+(\theta-N r \phi) \lambda \sin x
$$

The solutions of equations (1) to (4) are in the form of

$$
\psi=x f(x, y), \theta=\theta(x, y), \quad \phi=\phi(x, y)
$$

Substituting equation (8) into Equations (3), (4), and (7), the following boundary layer equations take the form of

$$
\begin{gathered}
\frac{\partial f}{\partial y}=[1+(\theta-N r \phi) \lambda] \frac{\sin x}{x} \\
\frac{\partial^{2} \theta}{\partial y^{2}}+f \frac{\partial \theta}{\partial y}+N b \frac{\partial \theta}{\partial y} \frac{\partial \phi}{\partial y}+N t\left(\frac{\partial \theta}{\partial y}\right)^{2}=x\left(\frac{\partial f}{\partial y} \frac{\partial \theta}{\partial x}-\frac{\partial f}{\partial x} \frac{\partial \theta}{\partial y}\right) \\
\frac{\partial^{2} \phi}{\partial y^{2}}+L e f \frac{\partial \phi}{\partial y}+\frac{N t}{N b} \frac{\partial^{2} \theta}{\partial y^{2}}=x L e\left(\frac{\partial f}{\partial y} \frac{\partial \phi}{\partial x}-\frac{\partial f}{\partial x} \frac{\partial \phi}{\partial y}\right)
\end{gathered}
$$

The boundary conditions become

$$
\begin{aligned}
& f(x, y)=0, \theta^{\prime}(x, y)=-\gamma(1-\theta(x, y)), \quad \phi(x, y)=1 \quad \text { at } y=0, \\
& \theta(x, y) \rightarrow 0, \phi(x, y) \rightarrow 0 \quad \text { as } y \rightarrow \infty
\end{aligned}
$$

The regular fluid $\left(N_{b}=N_{t}=N_{r}=0\right)$ for the mixed convection problem over a 
horizontal circular cylinder are described by

$$
\begin{gathered}
\frac{\partial f}{\partial y}=[1+\theta \lambda] \frac{\sin x}{x} \\
\frac{\partial^{2} \theta}{\partial y^{2}}+f \frac{\partial \theta}{\partial y}=x\left(\frac{\partial f}{\partial y} \frac{\partial \theta}{\partial x}-\frac{\partial f}{\partial x} \frac{\partial \theta}{\partial y}\right) \\
f(x, 0)=0, \quad \theta^{\prime}(x, 0)=-\gamma(1-\theta(x, 0)), \theta(x, \infty)=0
\end{gathered}
$$

Near the lower stagnation point, $x \approx 0$, equations (13) to (15) for the case of mixed convection reduce to the following ordinary differential equations

$$
\begin{array}{r}
f^{\prime}=1+(\theta-N r \phi) \lambda \\
\theta^{\prime \prime}+f \theta^{\prime}+N b \theta^{\prime} \phi^{\prime}+N t \theta^{\prime \prime}=0 \\
\phi^{\prime \prime}+" L e f \phi^{\prime}+\frac{N t}{N b} \theta^{\prime \prime}=0
\end{array}
$$

subject to boundary condition

$$
\begin{aligned}
& f(0)=0, \quad \theta^{\prime}(0)=-\gamma(1-\theta(0)), \quad \phi(0)=1 \quad 0 \\
& \theta(0) \rightarrow 0, \quad \phi(0) \rightarrow 0
\end{aligned}
$$

where primes denote the differentiation with respect to $y$. It is also worth highlighting at this point that when $\gamma \rightarrow \infty$, the convective boundary condition reduced to constant wall temperature as solved by Tham et. al [9].

\section{Results and discussion}

The ordinary differential equations (16) to (18) with corresponding boundary conditions (19) were solved numerically. Numerical investigation of the boundary problem has been carried out for different values of parameters; convective parameter $\gamma$ and Lewis number $L e$ using Keller-box method.

Table 1. Values of the heat coefficient $-\theta^{\prime}(0)$ when $N_{b}=0, N_{t}=0, N_{r}=0, L e=0$ and $\gamma \rightarrow \infty$

\begin{tabular}{|c|c|c|c|}
\hline$\lambda$ & \multicolumn{3}{|c|}{$-\theta^{\prime}(0)$} \\
\cline { 2 - 4 } & Cheng [13] & Tham et. al [9] & Present result \\
\hline 0.0 & 0.7980 & 0.7979 & 0.7973 \\
\hline 0.5 & 0.9157 & 0.9156 & 0.9146 \\
\hline 1.0 & 1.0192 & 1.0191 & 1.0178 \\
\hline 2.0 & 1.1988 & 1.1987 & 1.1968 \\
\hline
\end{tabular}

For the validation purposes, we solved the case at the lower stagnation point $x \approx 0$ of the cylinder. Comparison has been made with data produced by Cheng [13] and Tham et. al [9] where $\gamma \rightarrow \infty$ (CWT) for the values of heat transfer coefficient $-\theta(0)$ as provided in table 1 . The comparison between these results is found to be good, based on the obtained numerical result.

Our aim is to observe the influence of the governing parameters such as $\gamma$ and $L e$ on the heat and mass flow characteristics. Also, the results are presented graphically for the effect of the above parameters. Fig.2 illustrates the behaviour of velocity profile $f^{\prime}(y)$ with changes in the values of $\gamma$ and Le parameter. In fig.2(a), increasing $\gamma$ produces an increase 
in velocity. Further, increasing the values of $L e$ in fig. 2(b) are seen to impact on acceleration of the flow represented by the increases in the velocity profile.

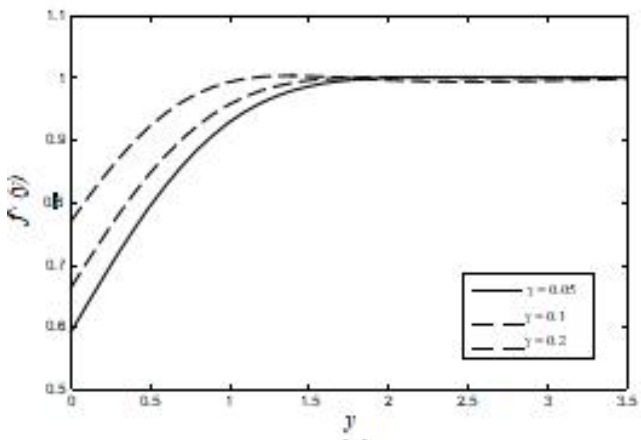

(a)

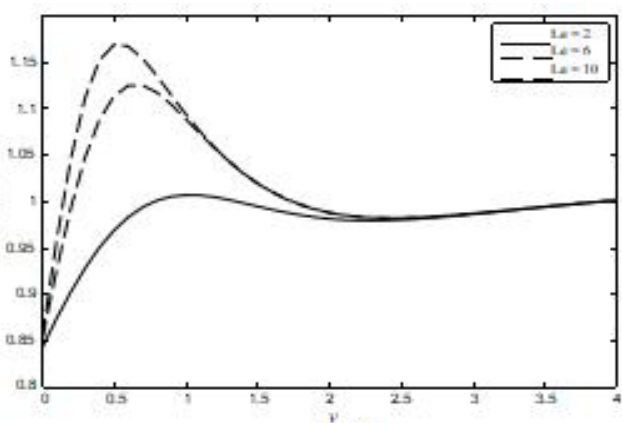

(b)

Fig. 2. Velocity profile $f^{\prime}(y)$ for various values of $\gamma$ and $L e$.

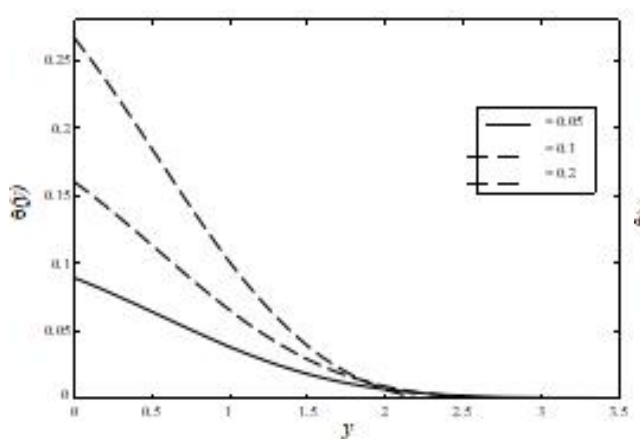

(a)

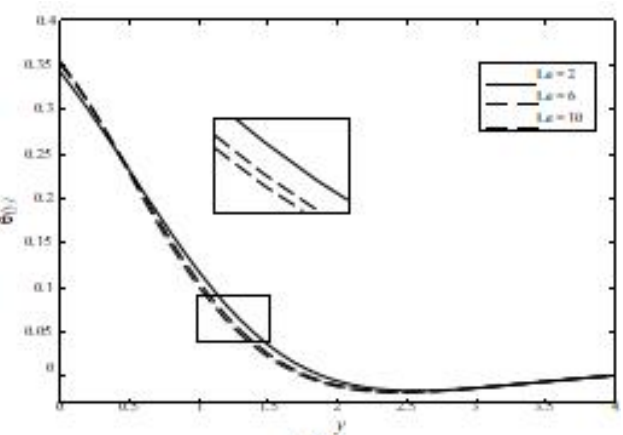

(b)

Fig. 3. Temperature profile $\theta(y)$ for various values of $\gamma$ and $L e$.

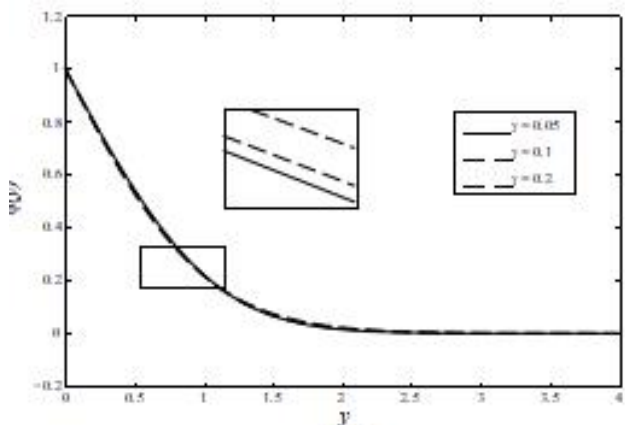

(a)

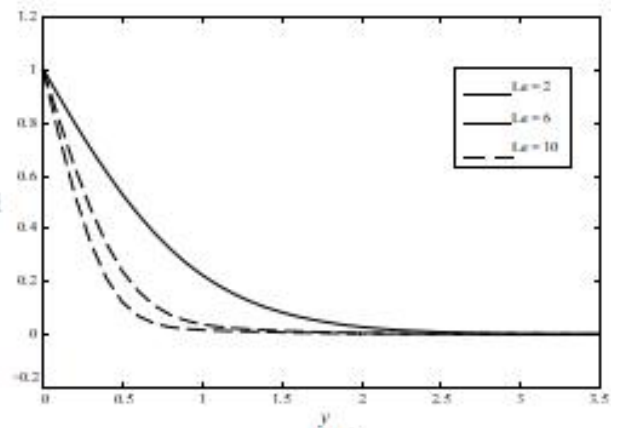

(b)

Fig. 4. Nanoparticle volume fractions profile $\phi(y)$ for various values of $\gamma$ and $L e$.

The effect of $\gamma$ and $L e$ on temperature profile $\theta(y)$ is demonstrated in fig. 3. Increasing the Lewis number produce a decrease in temperature profile (fig.3(b)). However the opposite trend were observed for $\gamma$ as illustrated in fig.3(a). Fig. 4(a) and (b) presents an effect of $\gamma$ and $L e$ on the nanoparticle volume fraction $\phi(y)$, respectively. From these figures, the nanoparticle volume fraction $\phi(y)$ decrease as $L e$ increases. This is due to the increase of buoyancy induced flow along the surface. Conversely, different result is observed for $\gamma$. 


\section{Conclusion}

This study demonstrates the effect of governing parameters on the mixed convection over a horizontal circular cylinder embedded in porous medium saturated by nanofluid. We looked into the effects of $\gamma$ and $L e$ on the flow and heat transfer characteristic. Therefore, from the results, the following conclusions are presented:

- increasing the $\gamma$ produce an increases in all profile; velocity, temperature and nanoparticle volume fractions profile.

- velocity increase when Le increases, however different patterns are observed for the temperature and nanoparticle volume fractions profile.

\section{Acknowledgement}

The authors would like to acknowledge the financial supports received in the form of the research university grants (RDU170358 and RDU1703187) from the Universiti Malaysia Pahang.

\section{References}

1. Tiwari R K and Das M K 2007 International Journal of Heat and Mass Transfer 50 $2002-2018$

2. Buongiorno J 2006 Journal of Heat Transfer 128 240-250

3. Nield D A and Kuznetsov A V 2009 International Journal of Heat Mass Transfer 52 5792-5795

4. Cheng P and Minkowycz W J 1977 Journal of Geophysical Research 82 2040-2044

5. Nield D A and Kuznetsov A V 2011 International Journal of Heat Mass Transfer 54 374-378

6. Kuznetsov A and Nield D 2011 International Journal of Thermal Sciences 50 712-717

7. Khan W and Aziz A 2011 International Journal of Thermal Sciences 50 2154-2160

8. Hussanan A, Khan I, Hashim H, Mohamed M A, Sarif N M and Salleh M Z 2016 Jurnal Teknologi 78121 - 126

9. Tham L, Nazar R and Pop I 2014 International Journal of Thermal Sciences 8421 33

10. Sarif N M, Salleh M Z and Nazar R 2016 ARPN Journal of Engineering and Applied Sciences 11 7274-7278

11. Na T Y 1979 Computational Methods in Engineering Boundary Value Problems (New York: Academic Press)

12. Cebeci T and Bradshaw P 1988 Physical \& Computational Aspects of Convective Heat Transfer (New York:Springer)

13. Cheng P 1982 International Journal of Heat and Mass Transfer 25 1245-1246 